\title{
Visión e integración de la perspectiva ambiental en la Universidad Indígena de Bolivia - UNIBOL
}

\section{Visão e integração da perspectiva ambiental na Universidade Indígena da Bolívia - UNIBOL}

\section{Vision and integration of the environmental perspective of the Indigenous University of Bolivia - UNIBOL}

\author{
Germán Vargas Callejas ${ }^{1}$
}

\begin{abstract}
RESUMEN
La sostenibilidad ambiental es uno de los objetivos de las universidades, que en función de sus recursos y singularidades económicas y socioculturales, paulatinamente, están asumiendo este desafío. En el contexto boliviano, en 2008, inician sus labores las universidades indígenas, cuya creación, funcionamiento y proyección se basan en principios ambientales propios de las culturas Aymara, Quechua y Guaraní, que a través de estas instituciones académicas pretenden conservar, transmitir y actualizar sus saberes y modelos de desarrollo, que tienen como referente fundamental la convivencia respetuosa y equilibrada con la naturaleza.
\end{abstract}

Palabras clave: Educación Ambiental; sostenibilidad; vivir bien; conocimiento indígena; Universidades Indígenas.

DOI: $10.1590 / 0104-4060.38109$

1 Grupo de Investigación en Pedagogía Social y Educación Ambiental (SEPA). Universidad de Santiago de Compostela, Facultad de Ciencias de la Educación, Departamento de Teoría de la Educación, Historia de la Educación y Pedagogía Social (Campus Vida), 15782, Santiago de Compostela, España. 


\title{
RESUMO
}

A sustentabilidade ambiental é um dos objetivos das universidades que, em função de seus recursos e singularidades econômicas e socioculturais, paulatinamente, estão assumindo este desafio. No contexto boliviano, em 2008, as universidades indígenas iniciam seus trabalhos, cuja criação, funcionamento e projeção se baseiam em princípios ambientais próprios das culturas Aimará, Quéchua e Guarani, que através destas instituições acadêmicas pretendem conservar, transmitir e atualizar seus saberes e modelos de desenvolvimento, que têm como referência fundamental a convivência respeitosa e equilibrada com a natureza.

Palavras-chave: Educação Ambiental; sustentabilidade; viver bem; conhecimento indígena; Universidades Indígenas.

\begin{abstract}
The environmental sustainability is one of the main goals of universities that according to their economic, social and cultural resources and singularities are gradually assuming this challenge. In 2008, Bolivian Indigenous universities started their efforts based on the environmental principals of the Aymara, Quechua and Guarani cultures. Through these academic institutions, they expect to conserve, to pass on and to update their knowledge and models of development maintaining the respectful and balanced relation with Nature as a fundamental basis.
\end{abstract}

Keywords: Environmental Education; sustainability; living well; Indigenous knowledge; Indigenous Universities.

\section{Introducción}

La sostenibilidad es el horizonte que, a pesar de las críticas (NAREDO, 1997; MEIRA, 2006; TAIBO, 2014), guía la labor de las universidades que se comprometen con el cuidado del medio ambiente y la promoción de un modelo de vida menos nocivo para el hombre y la naturaleza. En Bolivia, algunos centros de educación superior han optado, aunque de forma incipiente, por la vía de la sostenibilidad. Una orientación del desarrollo que está definida por la cultura local, cuyas singularidades condicionan e imprimen unas características propias a este proceso que, en términos tradicionales, coincide con el "buen vivir". Un concepto cuyas raíces prenden en las prácticas cotidianas y el horizonte utópico de la vida buena de los indígenas quechuas, aymaras, guaraníes. 
Considerando las características culturales del Estado Plurinacional de Bolivia y tomando como objeto de estudio la Universidad Indígena de Bolivia (UNIBOL), el objetivo del presente artículo es describir y analizar cómo se integra la perspectiva ambiental en el proceso de creación y funcionamiento de este centro académico, fundado específicamente para salvaguardar y, al mismo tiempo, promocionar la cultura y el conocimiento de los pueblos indígenas.

La realización de este artículo ha supuesto, a nivel metodológico, la revisión de 200 artículos de los medios de prensa más destacados de Bolivia, Los Tiempos, Opinión, La Razón, El Deber, El Diario, El Correo del Sur y Cambio, durante el período comprendido entre la propuesta de creación de esta entidad académica en 2008, hasta el presente. Una tarea que ha sido complementada con el análisis de la Constitución Política del Estado Plurinacional de Bolivia y la Ley de Educación “Avelino Siñani - Elizardo Pérez”, además de la revisión de las publicaciones más relevantes en torno a este tema, contenidas en libros y revistas especializadas y de divulgación, cuyo contenido ha sido estudiado e integrado en función de las categorías de análisis previstas para la elaboración de este documento.

\section{Las universidades sostenibles}

Los centros de educación superior, por su función docente e investigadora, influyen en los procesos de cambio, en cuanto son responsables de aportar los conocimientos necesarios para dar respuesta a los problemas y las demandas sociales; máxime cuando uno de sus mayores compromisos estriba en la formación de los futuros profesionales, de cuya labor dependerá, en gran medida, el proceso de construcción social. Por tanto, las universidades no existen al margen de las circunstancias del entorno, como el resto de la sociedad deben enfrentar y dar respuesta, por ejemplo, a las dificultades derivadas de la crisis ambiental global (DECLARACIÓN DE TALLAROIS, 1993).

Conscientes de su responsabilidad, desde finales de los años 60 del siglo pasado, algunas universidades han iniciado procesos específicos para responder a los desafíos que propone la crisis ambiental (ALBA, 2006), desarrollando actuaciones que pretenden minimizar el impacto humano en la destrucción de la naturaleza. Un compromiso que se profundiza en los años 90 debido al incremento de la preocupación mundial por los problemas ambientales, que se hace visible en declaraciones como la de Talloires en 1990, de Halifax en 1991, Lüneburg en 2001, la realización de la Cumbre de la Tierra en 1992 y la creación 
de redes y asociaciones de universidades sostenibles como COPERNICUS (Red de cooperación entre universidades europeas para el intercambio de conocimientos y experiencias sobre el desarrollo sostenible) en 1993 y la Global Higher Education Partnership for Sustainability (GHEPS) establecida en el año 2001.

En la mayoría de las universidades el concepto de la sostenibilidad ha sido introducido en base a una estructura que contempla tres ejes: el primero, relacionado con la generación o producción de conocimiento que contempla aspectos como la incorporación de los temas ambientales a los currículos formativos a través del diseño de titulaciones ad doc (Ciencias Ambientales, Ingeniería Ambiental, Doctorado en Educación Ambiental...) o, en su caso, el diseño de asignaturas específicas (Educación Ambiental, Pedagogía Ambiental...), y la integración especializada o transversal de las cuestiones ambientales en los procesos de investigación básica y aplicada, proceso que en general se conoce como ambientalización curricular y de la investigación; el segundo, la divulgación, sensibilización y generación de dinámicas participativas en torno a la problemática ambiental, que es realizada a través de múltiples proyectos y actividades (conferencias, jornadas, cursos de verano, exposiciones, voluntariado...), orientados a sensibilizar y concienciar a la comunidad universitaria, para generar un nuevo marco de valores y competencias respetuosas frente a la naturaleza; el tercero, de carácter más burocrático y de organización del entorno y los recursos, hace referencia a la planificación y gestión ambiental, cuyo objetivo es optimizar los recursos, maximizar los beneficios y reducir los riesgos y problemas ambientales en el ámbito universitario, en este nivel se integran los proyectos de ordenación urbana, la gestión de los residuos y de la energía, las evaluaciones de calidad ambiental, la promoción de la movilidad sostenible y la medición del impacto ambiental - huella ecológica - de la institución y sus actividades en el medio ambiente (AZNAR; ULL, 2009).

Según Alba (2006) para encarrilar a una institución de educación superior en la vía de la sostenibilidad es preciso seguir algunas pautas de actuación: 1) el establecimiento de un compromiso institucional y una visión estratégica de sostenibilidad de la universidad; 2) desarrollar una estructura que asegure el compromiso y la actuación dentro del gobierno de la universidad; 3) fomentar y consolidar en compromiso de base de la comunidad universitaria; 4) desarrollar y mantener un servicio técnico que asegure el desarrollo de las políticas de sostenibilidad; y, por último, 5) institucionalizar los resultados, estableciendo procedimientos de seguimiento y evaluación de la sostenibilidad en la universidad. Aspectos que en mayor o menor medida son observados por las universidades comprometidas con la sostenibilidad. 


\section{Emergencia y consolidación de las Universidades indígenas en Bolivia}

El sistema Universitario Boliviano no se ha desarrollado al margen de las iniciativas de sostenibilidad promovidas en el ámbito internacional, en todo caso, su integración en este movimiento, hasta ahora, ha sido marginal. No obstante, es innegable que algunas instituciones académicas si están empeñadas en implementar la perspectiva de la sostenibilidad desde un enfoque propio, que difiere de los planteamientos internacionales elaborados para el establecimiento de las universidades sostenibles, en cuanto se fundamenta en cuestiones de carácter cultural y político de profundo calado, ligados a procesos políticos de construcción nacional y, al mismo tiempo, a una cosmovisión cultural que tiene como referente central a la naturaleza.

Considerando como caso de estudio a los tres centros académicos que integran UNIBOL (Universidad Indígena de Bolivia, comunitaria, intercultural y productiva), creada el 2 de agosto de 2008 a través del Decreto Supremo 29.664 y reconocidas en la Ley educativa de Bolivia como

[...] instituciones académico científicas de carácter público, articuladas a la territorialidad y organización de las naciones y pueblos indígenas originario campesinos del Estado Plurinacional, que desarrollan formación profesional e investigación, generan ciencia, tecnología e innovación a nivel de pre grado y post grado (LEY DE LA EDUCACIÓN “AVELINO SIÑANI - ELIZARDO PÉREZ”, Artículo 60, inciso 1).

Se observa que su fundación reposa en tres ejes: la historia de lucha por la valoración y recuperación de las culturas indígenas (CHOQUE, 2012); un marco legislativo ambientalista y, con un sentido transversal, la intención política de construir un nuevo orden de convivencia democrática que tome en cuenta la diversidad cultural y biológica.

La UNIBOL dependiente de los Recursos del Fondo Indígena provenientes del Impuesto Directo a los Hidrocarburos, se ha constituido como una entidad académica que dispone de tres centros autónomos: 1) la Universidad Indígena Tupac Katari, situada en la comunidad indígena de Warisata, en el departamento de La Paz, institución donde se imparten, en lengua aymara y castellano, las carreras de Agronomía Altiplánica, Industria de Alimentos, Industria Textil, Veterinaria y Zootecnia; 2) la Universidad Indígena Casimiro Huanca, ubicada en el departamento de Cochabamba, en la zona tropical de Chimoré, colonizada 
en su mayoría por indígenas quechuas, forma a los estudiantes en Agronomía Tropical, Industria de Alimentos, Forestal, Piscicultura; 3) la Universidad Indígena Apiahuayky Tumpa, localizada en Chuquisaca, en el poblado nativo de Curuyuqui, donde se enseña en lengua guaraní y castellano las carreras de Hidrocarburos, Forestal, Piscicultura, Veterinaria y Zootecnia. Estos tres centros otorgan títulos de técnico superior, licenciatura y maestría. Todos los estudiantes para titularse deben presentar una tesis en uno de los idiomas nativos, en función de la ubicación cultural del centro.

Las universidades indígenas, que están en funcionamiento a partir de 2009, además de la oferta curricular propia de cada carrera, también y de manera obligatoria en todas las titulaciones ofrecen materias transversales de Ciencias Sociales, Historia y Economía y Medio ambiente. Incidiendo en una formación integral que toma en cuenta los procesos de construcción histórica y cultural del país y, al mismo tiempo, la relación del desarrollo económico con la explotación sostenible de los recursos naturales. En consecuencia, estas universidades surgen con la finalidad de cumplir objetivos académicos, culturales, políticos y económicos.

A nivel académico, de producción y difusión del conocimiento, se pretenden descolonizar el pensamiento, tarea que en palabras de Eschenhagen (2014) se entiende como la revisión sistemática de los conceptos de análisis científico y una refundamentación de las bases epistemológicas empleadas para estudiar y comprender la realidad. A esto se suma el propósito de descolonizar el conocimiento, en la línea de promover el saber indígena como sustento de los procesos de construcción social.

Los objetivos culturales centrados en revitalizar, reconstruir y desarrollar las culturas indígenas, además de promocionar el diálogo de saberes, están estrechamente ligados a la idea de descolonización cultural, que implica poner en valor la producción de los diversos pueblos de Bolivia para generar nuevas bases de transmisión y construcción ideológica y cultural. Una tarea que necesariamente debe fundamentarse en los relatos y realidades locales, singularizadas por la cosmovisión de los pueblos indígenas y su principio de respeto y convivencia con la naturaleza. En términos del propio Viceministro de Educación Superior de Bolivia, Diego Pary, el objetivo de UNIBOL, entre otros, es “[...] reconstruir las identidades indígenas, desarrollar conocimientos científicos, saberes y tecnologías, orientadas por criterios comunitarios bajo principios de complementariedad, trabajo cooperativo, responsabilidad individual y colectiva, además del equilibrio con la naturaleza" (PRENSA BOLIVIANA: OPINIÓN, 02/08/2009).

A nivel económico, el objetivo de estas universidades es promocionar la iniciativa productiva y aportar al desarrollo económico nacional. UNIBOL 
debe servir para transformar las condiciones concretas de desarrollo del país, generando dinámicas productivas ajustadas a los principios de respeto a la naturaleza. En esta línea, indica Diego Pari (PRENSA BOLIVIANA: EL DIARIO, 25/03/2009) que las universidades indígenas tienen la obligación de formar profesionales que respondan a la realidad local y a las demandas de las comunidades, municipios y provincias.

En un sentido más transversal, entre los objetivos políticos destaca la intención de crear una base de conocimiento que justifique y legitime al Estado Plurinacional, además de formar indígenas para el ejercicio del poder gubernamental local y nacional. La universidad y su función están ligadas a procesos de construcción de ciudadanía, que implica educar personas capaces de ejercer sus derechos y obligaciones (VARGAS, 2006), además de proporcionar cuadros dirigentes preparados para la producción, difusión y ejercicio de la ideología que fundamenta el nacimiento de estas casas de estudio, en este caso, el indigenismo.

Roberto Aguilar, Ministro de Educación de Bolivia, considera que en términos globales de lo que se trata es de recuperar los saberes, el conocimiento, la cultura y las formas de organización de los pueblos indígenas originarios con la incorporación de la interculturalidad y el bilingüismo (PRENSA BOLIVIANA: OPINIÓN, 06/04/2009), generando las oportunidades formativas y de investigación que posibiliten la pervivencia de la cultura y de su medio de desarrollo, es decir, el entorno natural y social en el que se integran las comunidades indígenas. De forma coincidente, en el II Congreso de la Red de Universidades Indigenas, Interculturales y Comunitarias de Abya Yala, celebrado en agosto de 2011, se señala que los centros de formación superior indígenas deben priorizar: 1) la investigación en base a las necesidades y demandas de las comunidades y pueblos indígenas, que deben ser considerados como los principales actores de este proceso; 2) resguardar los conocimientos de los pueblos originarios que se están perdiendo; 3) incentivar la formación productiva y 4) insistir en el uso y recuperación de las lenguas nativas.

Para el cumplimiento de los objetivos propuestos, la UNIBOL tiene una estructura diferente al de las universidades convencionales, básicamente porque se fundamenta en un principio comunitario, que exige corresponsabilidad entre la universidad y la comunidad. En este sentido, la comunidad propone una lista de personas que deberían ser matriculadas en las distintas titulaciones. Durante el desarrollo de su formación los estudiantes deben rendir cuentas de sus resultados académicos a la sociedad local. El control sobre el funcionamiento de la universidad y sus miembros es ejercido por las Juntas Comunitarias integradas por los estudiantes, la Confederación Única de Trabajadores Campesinos de Bolivia (CSUTCB), la Confederación de Indígenas del Oriente Boliviano (Cidob), el Consejo Nacional de Ayllus y Marqas de Qullasuyu (Conamaq), 
la Confederación Nacional de Mujeres Campesinas Indígenas Originarias de Bolivia - Bartolina Sisa (CNMCIOB-BS) y la Confederación Sindical de Comunidades Interculturales (CSCIB).

A pesar del fuerte anclaje de las universidades indígenas en la cultura y los principios de vida de las comunidades nativas de Bolivia, estos centros no se erigen como espacios de negación del conocimiento científico occidental, en términos de Benecio Quispe, Viceministro de Educación Superior,

[...] esto no significa, como algunos quieren entender, que hay que negar lo que viene de Europa y Norteamérica, no se trata de negar, sino de lograr una complementariedad lógica diferente, cómo desde la academia se puede contribuir al proceso de descolonización y desneoliberalización como superación de la estructura colonial y neoliberal (PRENSA BOLIVIANA: CAMBIO, 13/01/2013).

Por tanto, contribuir a un proceso de cambio que responda a la cultura y los intereses locales, sobre la base de una lógica económica y social menos mercantilista.

La apuesta por un diálogo intercultural y el intercambio de saberes se visibiliza en la oferta formativa de estas universidades que, por ejemplo, si bien parten del principio de educar a sus estudiantes en lengua nativa, no niegan el aprendizaje y uso de idiomas como el castellano y el inglés, lengua última que es frecuentemente reclamada por el Presidente Evo Morales como un vehículo de comunicación que facilita la instrucción global de los universitarios, sobre todo indígenas. Otra singularidad de estas universidades es su orientación productiva, que propone el cambio social en base a prácticas socioeconómicas concretas, que exigen adecuar las titulaciones a las necesidades y posibilidades locales de desarrollo, un propósito que coincide con la construcción de un modelo económico fundamentado en el respeto a la naturaleza, en otros términos, basado en los principios del decrecimiento y la sostenibilidad.

Sintetizando lo expuesto, las universidades indígenas en Bolivia fundamentan su origen y funcionamiento en tres ejes sustantivos: la educación descolonizadora, la educación productiva y la educación comunitaria (CHOQUE, 2012), aspectos que también coinciden, como se observará más adelante, con las propuestas legislativas de la Constitución Política del Estado Plurinacional de Bolivia y su Ley de Educación. 


\section{Fundamentos histórico-culturales, epistemológicos y legislativos pro-ambientales de las universidades indígenas}

Las universidades indígenas se caracterizan por su forma singular de integrar los principios ambientales en su estructura y funcionamiento. En Bolivia, por las particularidades históricas, culturales y epistemológicas de las comunidades nativas, estas iniciativas nacen y se fundamentan en una cosmovisión, una ideología y una legislación pro-ambientalista, siendo el respeto y el cuidado de la naturaleza factores constitutivos de su propia esencia y no, como sucede en otras universidades convencionales, una parte estanca viabilizada a través de un programa, un proyecto o acciones aisladas.

Las bases epistemológicas del conocimiento indígena descansan en su concepción compleja de la vida, que se expresa en los vínculos de los seres humanos con sus semejantes y con la naturaleza en una doble dimensión: material y espiritual (SAN MARTÍN, 1997). Entre las culturas nativas está arraigada, en el presente con un carácter más marginal, la idea de interacción entre los seres de la naturaleza y las divinidades, por la cual nada ni nadie habita al margen de la presencia y la influencia de los demás seres, como ninguno construye su parcela vital de forma solitaria: "todo" y "todos" participan en este proceso, estructurando una realidad compleja que sólo es comprensible desde una percepción global y holística (VARGAS, 2005). Por tanto, no es sólo el sujeto humano quien transforma el mundo, en esta acción también se involucran otros entes naturales y sobrenaturales, cuya presencia condiciona la construcción de un estilo de vida y de desarrollo.

La comprensión de la naturaleza como una totalidad dotada de conciencia y existencia autónoma constituye el fundamento cultural primordial de los pueblos indígenas. Una visión que condiciona los procesos de comunicación, la generación de conocimientos, la convivencia, la transformación material del entorno, en síntesis, toda la vida y su desarrollo. Considerando esta idea cardinal es correcto afirmar que la constitución de las universidades indígenas, en gran medida, responde al interés por revitalizar, consolidar y promocionar, a través del diálogo de saberes y la validación científica, la visión milenaria de convivencia respetuosa y recíproca entre el ser humano y la naturaleza.

Las coordenadas propias de las culturas nativas definen las características y la perspectiva epistemológica que debe fundamentar los procesos de enseñanza y aprendizaje en las universidades indígenas. Según la Declaración del Segundo Encuentro Internacional de Universidades Indígenas, llevado a cabo entre el 5 y 6 de agosto de 2009, en Quito, el desarrollo científico, cultural se fundamenta: 
[...] en el respeto a nuestros principios y valores de la Cosmovisión Andina, como es la vitalidad, dualidad, complementariedad, armonía, equilibrio, la hermandad, reciprocidad y convivencia entre nosotros mismos y con otras sociedades y culturas. Consideramos como un aporte al conocimiento universal, las diversas cosmovisiones indígenas que permiten a los pueblos y nacionalidades indígenas, mirar de manera diferente la vida, el trabajo, la educación, la familia, la espiritualidad que garantiza el buen vivir para todos y todas. Consideramos a los pueblos y nacionalidades en la capacidad de generar nuevos paradigmas, epistemológicos, ciencias y tecnologías para vivir en equilibrio con la madre tierra (ENCUENTROS, 2009, p. 2).

Es innegable que en el presente estamos asistiendo a una transformación en los paradigmas que explican y fundamentan lo que es o debería de ser el conocimiento científico (SANTOS, 2000; BARBETA, 2012), debido a la irrupción de otros modos de generar saberes y a la conciencia de la existencia de otros sistemas de conocimiento que siguen pautas diferentes al método científico occidental. Procesos de construcción del conocimiento que hasta hace poco eran reconocidos, en términos peyorativos, como: "conocimiento tradicional", "saber popular", "conocimiento local", "conocimiento del pueblo", "cultura popular" y, en algunos casos, como "ciencia tradicional" para diferenciarla de la ciencia comprendida en términos cartesianos.

Un análisis epistemológico del saber indígena lleva a establecer que, por ejemplo en el contexto quechua-andino, el conocimiento se caracteriza por tener rasgos propios en múltiples niveles: primero, de generación y transmisión de los saberes; segundo, en cuanto a los agentes que participan en el proceso de producción y justificación de los conocimientos; tercero, relacionado con el sentido y la utilidad de los saberes; y, cuarto, por el marco conflictivo, inter-generacional e intercultural, en el cual se desarrolla el saber indígena. Siguiendo los argumentos de Emery et al. (1997), Vargas (2005) y Cayuqueo (2007), a continuación se destacan los principales rasgos distintivos de esta forma de conocimiento, que fundamenta la propuesta epistemológica de las universidades indígenas.

La mayoría de los autores (HAVERKORT et al., 2003; KING y SCHIELMANN, 2004; GROS y MIGUEL, 2010) destaca que el conocimiento indígena se basa en la experiencia práctica, en cuanto resulta de la sistematización de las vivencias sentidas y percibidas a lo largo de la historia, que han ido cristalizando a través de la aplicación sistemática y repetitiva en prácticas que, para su mantenimiento y generalización en la comunidad local, han sido encumbradas al estatus de "saber tradicional". Un conjunto de conocimientos anclados a un territorio y propios de un colectivo que ha desarrollado los mecanismos 
pertinentes para su generación, transmisión y aplicación. En consecuencia, el conocimiento indígena no es especulativo, es un saber validado en la vida misma, transmitido y conservado por su utilidad para solucionar problemas cotidianos de toda índole (SANTOS, 2000).

Los saberes que circulan en el entorno indígena no son transmitidos de forma indiscriminada, en realidad sólo se transfieren y cultivan aquellos saberes que tienen sentido y utilidad para la vida. Las estrategias de difusión del conocimiento se fundamentan en la vivencia práctica, la comunicación oral y por medio de actividades cotidianas en las cuales las generaciones menores van recibiendo el legado de los mayores a través de rituales específicos, adecuados al tipo de saber que se pretende potenciar, transmitir y conservar.

En las comunidades tradicionales no se ambiciona la generalización del conocimiento, es más, los saberes están íntimamente ligados a las circunstancias y condiciones del contexto social y natural. El ecosistema comunitario, el territorio próximo constituyen la base para el desarrollo y la aplicación del conocimiento; en ese entorno limitado se pretende el descubrimiento y la comprensión "de lo otro", a partir de las relaciones comunicativas cotidianas y el desentrañamiento de las leyes y fenómenos propios de la naturaleza. Efectivamente es un conocimiento delimitado y práctico, cuyo objetivo primordial es dar respuesta a las circunstancias, problemas y demandas locales, a partir de la aplicación de recursos y soluciones de carácter endógeno.

Por su arraigo en la vida cotidiana y su intención de dar respuesta a múltiples situaciones, el conocimiento indígena es holístico, de carácter social, material y espiritual (VARGAS, 2005). La confluencia de estas tres dimensiones, en su conjunto, sirve para explicar los fenómenos sociales y naturales, sin relegar a lo supranatural que en el contexto tradicional constituye el fundamento mismo de la existencia.

Otro rasgo propio del conocimiento indígena es que responde a una construcción colectiva. Los saberes desarrollados por cada generación son puestos a prueba en la vida de la comunidad y se van mejorando y acumulando en función de la comprobación colectiva. La vigencia de este saber no se sustenta en el poder de un individuo, sino en lo que marca la tradición, que como tal debe de ser aceptada por la mayoría de los componentes de la comunidad; en consecuencia, los cánones del conocimiento indígena son transformables siempre y cuando la colectividad experimente y asuma el cambio. Por esta particularidad, el saber tradicional sólo tiene utilidad y sentido si es compartido. En el aislamiento, en el trabajo solitario pierde significado y contenido, ya que desde una perspectiva endógena, el mundo sólo se puede explicar a partir de la experiencia comunal, sin comunidad se disgrega el conocimiento, sin una práctica concreta y su arraigo a un territorio específico el saber pierde valor y desaparece. 
La relación conocimiento, territorio, comunidad se magnifica debido al principio de reciprocidad, por el cual las personas aprenden y enseñan de forma cooperativa y compartida, con los miembros de su entorno social y natural más próximo, aquello que incide en la existencia colectiva (MCKINLEY; BRAYBOY; MAUGHAN, 2009). Para los indígenas el conocimiento que no se comparte carece de utilidad y significado; en esta línea, mantener y promocionar el bienestar en la comunidad implica colaborar y lograr que la mayoría interiorice y practique los conocimientos que garantizan la vigencia de la tradición y con ella la vida misma de la sociedad local.

Los mecanismos desarrollados para el cultivo, conservación y difusión del saber indígena son múltiples, destacan por su importancia los rituales, las fiestas y algunos elementos de la vida cotidiana ligados específicamente al trabajo agrícola. En el ámbito comunicativo, para la transmisión de saberes se emplean la palabra, el canto y la observación de las acciones individuales y comunales; el ejemplo, los rituales y todo elemento relacionado con la integración del hombre en la naturaleza (VARGAS, 2005). El conocimiento heredado y generado es difundido entre los miembros de la comunidad para dar lugar a estrategias adecuadas de integración en la tradición o bien para el desarrollo de conocimientos innovadores que, necesariamente, deben tener como referente al saber que mana de la tradición.

El conocimiento indígena, a pesar de su arraigo y justificación en la tradición, no es un saber obsoleto y fosilizado, más al contrario es dinámico, porque permite la integración de innovaciones y transformaciones que dan lugar a mejoras o adaptaciones en los múltiples ámbitos de la vida. Por otra parte, es un saber acumulativo, que resulta de la suma, en el tiempo, de saberes elaborados por diversas generaciones que, de manera lenta, han ido estructurando una base estable de conocimientos seleccionados y cultivados por su idoneidad para dar respuesta a las exigencias cambiantes del entorno vital, donde a pesar de la conciencia de cambio, existe una fuerte tendencia a mantener la estabilidad, siendo lento y pausado el proceso colectivo de introducción de nuevos saberes.

La complejidad del conocimiento indígena se hace visible en su capacidad para integrar las dimensiones materiales, sociales y espirituales del mundo y de la vida (SAN MARTÍN, 1997; VARGAS, 2005). Por tanto, este saber no responde de forma aséptica a la satisfacción de necesidades y a la solución de problemas, más al contrario estas respuestas deben estar justificadas por un ente supranatural y por su valor para el bienestar de la comunidad; en este sentido, el conocimiento vernáculo se fundamenta en un principio comunal, en cuanto atañe al bienestar de todos y en la correlación entre los productos de la razón y la vivencia espiritual. 
La complejidad del conocimiento indígena también se hace visible en esa relación intrínseca entre sujeto y objeto de conocimiento, proceso gnoseológico que considera inseparables al ser que conoce y la naturaleza a ser conocida. En la visión nativa de construcción del conocimiento la interdependencia se erige como un criterio axial, así, los humanos y su acción racional se integran durante el proceso cognitivo como parte constitutiva - cuerpo - de la naturaleza misma, una visión que difiere totalmente del saber occidental que insiste en la escisión entre el sujeto y el objeto de conocimiento (SANTOS, 2000; MARZO DE, 2010). En el mundo indígena, por su cosmovisión relacional-comunicativa, "todo" y "todos" participan en el proceso de construcción del conocimiento. Por tanto, no es el sujeto humano quien de forma exclusiva transforma la realidad, en esta tarea también participan otros agentes (naturales y sobrenaturales) cuya presencia comunicativa y educativa condiciona la emergencia de un modelo de vida.

En este complejo de interacciones, el ser humano se encuentra en una situación constante de enseñanza y aprendizaje, un proceso singularizado por su carácter compartido, racional, sensorial y mítico, por medio del cual aprehende las propiedades de su entorno y responde a sus exigencias. En consecuencia, la producción de conocimiento no resulta de una suma lineal de saberes, tampoco de la "iluminación" individual y mucho menos de una visión unidimensional de la realidad. La generación de conocimiento se presenta como un proceso intencionado, selectivo, sistemático y relacional (MCKINLEY; BRAYBOY; MAUGHAN, 2009), un acto cognoscitivo holístico e integral por el cual el indígena percibe su propia identidad, se reconoce como un sujeto situado en un tiempo y un espacio, y adquiere conciencia de su forma de ser y estar en el mundo.

Estas pautas epistemológicas indígenas que " [...] no conciben la fragmentación entre el ser humano y la naturaleza, coinciden en la necesidad de reconocer la importancia de la reciprocidad y el respeto por la vida y el entorno natural" (ESCHENHAGEN, 2014, p. 95). Claves que en síntesis constituyen el marco cultural e ideológico que justifica y legitima la fundación y funcionamiento de las universidades indígenas en Bolivia, cuya misión explicita es actualizar este marco de conocimientos y proponer una epistemología distinta de la tradición occidental, abriendo de este modo una brecha en la visión unilateral y eurocéntrica de hacer ciencia (BARBETA, 2012), una perspectiva que es reforzada por las declaraciones del Ministro de Educación de Bolivia, Roberto Aguilar, quien sostiene que en las universidades indígenas "[...] se proyectará una formación diferente para los nuevos profesionales por cuanto su concepción tiene una base consistente que proviene de las naciones y pueblos indígenas originarios" (PRENSA BOLIVIANA: LA RAZÓN, 12/04/2009).

Además de la base histórica-cultural y epistemológica, la implantación de las universidades indígenas también responde a un proyecto político del Estado 
Plurinacional de Bolivia. En la Constitución Política del Estado Plurinacional (CPE) y la Ley de Educación (LE) se manejan conceptos que permiten vislumbrar el propósito de promocionar una sociedad identificada por el respeto a todo tipo de vida, la convivencia comunitaria y el cuidado de la naturaleza, aspectos centrales en la creación, funcionamiento y proyección de estos centros académicos.

La orientación ambientalista de la Constitución se visibiliza por ejemplo cuando señala que " [...] las personas tienen derecho a un medio ambiente saludable, protegido y equilibrado. El ejercicio de este derecho debe permitir a los individuos y colectividades de las presentes y futuras generaciones, además de otros seres vivos, desarrollarse de manera normal y permanente" (CONSTITUCIÓN POLÍTICA DEL ESTADO PLURINACIONAL DE BOLIVIA, artículo 33). Un derecho que debe ser viabilizado a través de múltiples procesos, entre otros, la educación que

[...] tendrá como objetivo la formación integral de las personas y el fortalecimiento de la conciencia social crítica en la vida y para la vida. La educación estará orientada a la formación individual y colectiva; al desarrollo de competencias, aptitudes y habilidades físicas e intelectuales que vincule la teoría con la práctica productiva; a la conservación y protección del medio ambiente, la biodiversidad y el territorio para el vivir bien (CONSTITUCIÓN POLÍTICA DEL ESTADO PLURINACIONAL DE BOLIVIA, artículo 80, inciso I).

El anclaje a la perspectiva ambiental también se observa en los planteamientos de la Ley de Educación, que entre sus objetivos destaca:

[...] desarrollar una conciencia integradora y equilibrada de las comunidades humanas y la Madre Tierra que contribuya a la relación de convivencia armónica con su entorno, asegurando su protección, prevención de riesgos y desastres naturales, conservación y manejo sostenible considerando la diversidad de cosmovisiones y culturas. (Para) [...] formar una conciencia productiva, comunitaria y ambiental en las y los estudiantes, fomentando la producción y consumo de productos ecológicos, con seguridad y soberanía alimentaria, conservando y protegiendo la biodiversidad, el territorio y la Madre Tierra, para Vivir Bien (LEY DE LA EDUCACIÓN “AVELINO SIÑANI - ELIZARDO PÉREZ”, artículo 5, inciso 9 y artículo 5, inciso 12). 
Para la realización de esta visión ambientalista en las universidades, la Constitución señala que

[...] la educación superior desarrolla procesos de formación profesional, de generación y divulgación de conocimientos orientados al desarrollo integral de la sociedad, para lo cual tomará en cuenta los conocimientos universales y los saberes colectivos de las naciones y pueblos indígenas originario campesinos (CONSTITUCIÓN POLÍTICA DEL ESTADO PLURINACIONAL DE BOLIVIA, artículo 91, inciso I).

En una línea similar, la Ley de Educación apunta que las universidades indígenas " [...] desarrollan procesos de recuperación, fortalecimiento, creación y recreación de conocimientos, saberes e idiomas de las naciones y pueblos indígena originario campesinos, desde el espacio académico científico, comunitario y productivo" (LEY DE LA EDUCACIÓN “AVELINO SIÑANI - ELIZARDO PÉREZ", artículo 60, inciso 2).

La responsabilidad académica y social de las universidades indígenas está ligada a un marco axiológico cuyos valores elementales son la naturaleza y el buen vivir, en esta línea

[...] el Estado asume y promueve como principios ético-morales de la sociedad plural: ama qhilla, ama llulla, ama suwa (no seas flojo, no seas mentiroso ni seas ladrón) Cuma qamaña (vivir bien) ñandereko (vida armoniosa), tecko kavi (vida buena), ivi maraei (tierra sin mal) y qhapaj ñan (camino o vida noble) (CONSTITUCIÓN POLÍTICA DEL ESTADO PLURINACIONAL DE BOLIVIA, artículo 8, Inciso I).

Por medio de los cuales se pretende "[...] contribuir a la convivencia armónica y equilibrada del ser humano con la Madre Tierra, frente a toda acción depredadora, respetando y recuperando las diversas cosmovisiones y culturas" (LEY DE LA EDUCACIÓN “AVELINO SIÑANI - ELIZARDO PÉREZ”, artículo 4, inciso 5). Por tanto, estos centros académicos deben impartir una "[...] educación de la vida y en la vida, para Vivir Bien [...] en armonía con la Madre Tierra y en comunidad entre los seres humanos" (artículo 3, inciso 11).

El fin último de la educación y por tanto de las universidades indígenas es promover el logro del sumaj kausay - vivir bien (VARGAS; VARGAS, 
2010) o el suma qamaña "buen vivir", según el idioma de cada pueblo, que se plantea como "[...] un paradigma comunitario basado en la vida en armonía y el equilibrio con el entorno" (ESCHENHAGEN, 2014, p. 93), que implica “[...] vivir en armonía y equilibrio; en armonía con los ciclos de la Madre Tierra, del cosmos, de la vida y de la historia, y en equilibrio con toda forma de existencia" (HUANACUNI, 2010, p. 80). Estos principios suponen "[...] una idea de la vida y del desarrollo basada en la conciencia de utilizar de la naturaleza sólo lo necesario, para evitar dañar y perjudicar su reproducción, comprometiendo así también los derechos de las generaciones futuras" (MARZO DE, 2010, p. 157).

\section{Conclusiones}

Recuperando el objetivo de este artículo expresado en términos de describir y analizar cómo se integra la perspectiva ambiental en el proceso de creación y funcionamiento de las universidades indígenas, se concluye afirmando que la visión ambiental es transversal a todas la acciones desarrolladas por estos centros de educación superior, cuya creación se fundamenta en principios de respeto y cuidado de la naturaleza, así como en la búsqueda de una convivencia equilibrada entre todas las formas de vida. Esta afirmación se sustenta en el hecho de que la instauración de estas universidades responde al cumplimiento de lo estipulado en la Constitución Política del Estado Plurinacional de Bolivia, sancionada en febrero de 2009 y los objetivos propuestos por la Ley de Educación Avelino Siñani - Elizardo Pérez, del 20 de diciembre de 2010, textos legislativos que cuyo contenido ha sido desarrollado y discutido con antelación a la creación de las universidades indígenas.

La integración de la perspectiva de la sostenibilidad en las universidades indígenas difiere de los modelos aplicados en las universidades tradicionales de occidente, debido sobre todo a que su fundación responde a objetivos de carácter académico, cultural, económico y político ligados a la promoción del conocimiento indígena y al planteamiento de una epistemología y un modelo de desarrollo alternativos, singularizado por su anclaje en el respeto y cuidado de la naturaleza. Un principio que impregna todo el proceso de organización y funcionamiento de estas universidades, algo que no sucede en los centros académicos de corte occidental, en los cuales la cuestión ambiental es residual y está integrada con una visión limitada a la transversalidad o bien a la promoción de programas, proyectos e iniciativas aisladas. 
La perspectiva ambiental y su inclusión están condicionadas por las culturas nativas, en palabras del Director de Educación Superior de Universidades del Ministerio de Educación, Juan Carlos Carrasco, la universidad indígena pretende " $[\ldots]$ generar ciencia reconociendo y 'academizando' los saberes propios y cotidianos de cada pueblo" (PRENSA BOLIVIANA: OPINIÓN, 04/08/2011), un propósito que responde a otros paradigmas epistemológicos y a intereses que coinciden con la promoción cultural, política, económica y social de las comunidades indígenas, lo cual convierte a las universidades indígenas en plataformas para la recuperación y proyección del conocimiento y, en general, de la cultura Aymara, Quechua, Guaraní y demás pueblos que integran el Estado Plurinacional del Bolivia, cuyo punto de confluencia es la relación armoniosa y equilibrada con la naturaleza.

Los centros de formación indígena han sido creados con la intención de poner en valor el saber tradicional, que según el Viceministro de Formación Profesional del Ministerio de Educación, Diego Pari, serán complementados con la tecnología y los conocimientos desarrollados en otros países y culturas (PRENSA BOLIVIANA: EL CORREO DEL SUR, 12/04/2009). En consecuencia, se orientan a ampliar los horizontes de desarrollo y proyección de los productos culturales de las naciones originarias, para dar lugar a nuevos paradigmas de conocimiento y desarrollo que profundicen en los objetivos que guían, actualmente, las iniciativas de sostenibilidad en las universidades occidentales, colocando como eje para la producción de saberes a la naturaleza, propuesta que implica un cambio radical en la concepción misma de la vida.

El fin último que sustenta y proyecta el funcionamiento de las universidades indígenas es el logro de la vida buena, o vivir bien, que contempla la armonía $\mathrm{y}$ equilibrio entre los seres humanos y todas formas de vida, propiciando para todos una existencia en plenitud (HUANAKUNI, 2010), respetuosa de los ritmos y ciclos sociales, biológicos y cosmológicos. Una vida en plenitud que sólo puede tener lugar en el marco de la comunidad (PATZI, 2013) integrada en un proceso de convivencia armoniosa en la naturaleza. Una propuesta que va más allá de la visión de vivir mejor, puesto que no trata de acumular más y ser más en detrimento de los otros, sino de ser y estar bien con los otros y la naturaleza.

\section{REFERENCIAS}

ALBA, D. Análisis de los procesos de gestión y educación para la sostenibilidad en las universidades públicas española. 2006. Disponible en: <http://www.redfia.net.gt/ 
bibliotecavirtual $/$ ministerio $\% 20 \mathrm{de} \% 20$ ambiente $\% 20$ Espan $\% \mathrm{CC} \% 83 \mathrm{a} /$ tendencias/tendencias_paginas197_215.pdf $>$. Acceso en: 24/04/2014.

AZNAR, P.; ULL, M. A. La formación de competencias básicas para el desarrollo sostenible: el papel de la Universidad. Revista de Educación, número extraordinario, p. 219-237, 2009.

BARBETA, P. Ecologías de los saberes campesinos: más allá del epistemicidio de la ciencia moderna. Reflexiones a partir del caso del Movimiento Campesino de Santiago del Estero Vía Campesina. Buenos Aires: CLACSO, 2012. Disponible en: <http://bibliotecavirtual.clacso.org.ar/clacso/becas/20120717115616/PabloBarbetta.pdf $>$. Acceso en: $13 / 04 / 2014$.

CAYUQUEO, S. Conocimiento Indígena en la globalización. 2007. Disponible en: $<$ http://www.vialibre.org.ar/mabi/5-conocimiento-indigena-globalizacion.htm $>$. Acceso en: 04/04/2014.

CHOQUE, M. E. Políticas de Educación para Pueblos Indígenas, Originarios, Campesinos y Afrodescendientes en Bolivia. En: MATO, D. (Coord.). Educación Superior y Pueblos Indigenas y Afrodescendientes en América Latina. Normas, Políticas y Prácticas. Caracas: Instituto Internacional de la UNESCO para la Educación Superior en América Latina y el Caribe (IESALC-UNESCO), 2012. p. 139-176. Disponible en: <http://www.iesalc. unesco.org.ve/dmdocuments/diversidad_libro4.pdf>. Acceso en: 22/03/2014.

CONSTITUCIÓN POLÍTICA DEL ESTADO PLURINACIONAL DE BOLIVIA, Ley de 7 de febrero de 2009 .

CUMBRE CONTINENTAL DE LOS PUEBLOS Y LAS NACIONALIDADES INDÍGENAS DE ABYA YALA, II., 2004. Conocimientos indigenas y propiedad intelectual. Disponible en: $<$ http://www.cumbreindigenabyayala.org/ponencias/index.html $>$. Acceso en: $02 / 04 / 2014$.

DECLARACIÓN DE TALLAROIS. Declaración de Lideres de Universidades para un Futuro Sostenible, 1993. Disponible en: <http://www.ulsf.org/pdf/Spanish_TD.pdf>. Acceso en: 17/03/2014.

EMERY, A. et al. Guidelines for Environmental Assessments and Traditional Knowledge. Ottawa: Informe del "Centre for Traditional Knowledge of the World Council of Indigenous People”, 1997.

ENCUENTRO DE LAS UNIVERSIDADES INDÍGENAS, AFRODESCENDIENTES E INTERCULTURALES Y DE ABYA YALA, II, 2009. Declaración del Segundo Encuentro Internacional de Universidades Indigena. Quito, 2009. Disponible en: < http://virtualtic. mobi/universidad/index.php?view $=$ article $\&$ catid $=35 \% 3$ Aresoluciones $\& i d=70 \% 3$ Adeclaracion-del-segundo-encuentro-internacional-de-universidades-indigenas \&format=pdf \& option $=$ com_content $\&$ Itemid=88\&lang $=$ es $>$. Acceso en: 12/03/2014.

ESCHENHAGEN, M. ¿El "buen vivir” en las universidades?: posibilidades y limitaciones teóricas. Integra Educativa, v. VI, n. 3, p. 89-105, 2014. 
GROS, P.; MIGUEL, N. Conocimientos del Pueblo Mayangna sobre la convivencia del hombre y la naturaleza. Paris: UNESCO, 2010.

HAVERKORT, B. et al. (Eds.). Antiguas raíces, nuevos retoños. El desarrollo endógeno en la práctica. La Paz: Plural Editores, 2003.

HUANACUNI, F. Vivir Bien/Buen Vivir. Filosofía, políticas, estrategias y experiencias regionales. La Paz: Coordinadora Andina de Organizaciones Indígenas, CAOI, 2010.

KING, L.; SCHIELMANN, S. El reto de la educación indígena: experiencias y perspectivas. Paris: UNESCO, 2004.

LEY DE LA EDUCACIÓN “AVELINO SIÑANI - ELIZARDO PÉREZ”, nº 070, 20 de diciembre de 2010. Disponible en: <http://www.minedu.gob.bo/phocadownload/ Ley_reglamentos/ley_educacion_avelino $\% 20$ sinani-elizardo $\% 20$ perez_070.pdf $>$. Acceso en: 22/02/2014.

MARZÓ DE, G. Buen vivir para una democracia de la Tierra. La Paz: Plural, 2010.

MCKINLEY, B.; BRAYBOY, J.; MAUGHAN, E. Indigenous Knowledges and the Story of the Bean. Harvard Educational Review, v. 79, p. 1-21, Spring 2009.

MEIRA, P. A. Elogio a la Educación Ambiental. Trayectorias, año VIII, n. 20-21, p. 41-51, Ene.-Ago. 2006.

NAREDO, J. M. Sobre el origen, el uso y el contenido del término sostenible, 1997. Disponible en: <http://habitat.aq.upm.es/cs/p2/a004.html>. Acceso en: 06/05/2014.

PATZI, F. Dos concepciones contrapuestas de la ley Avelino Siñani-Elizardo Pérez. Revista Ciencia y Cultura, v. 17, n. 3, 2013. Disponible en: <http://www.scielo.org.bo/scielo. php?pid=S2077-33232013000100004\&script=sci_arttext $>$. Acceso en: 30/04/2014.

SAN MARTÍN, J. Uk”amäpi. Así nomás es pues. Cochabamba: AGRUCO; Plural Ediciones, 1997.

SANTOS, B. Crítica de la razón indolente. Contra el desperdicio de la experiencia. Bilbao: Editorial Desclée de Brouwer, 2000.

TAIBO, C. ¿Por qué el decrecimiento? Un ensayo sobre la antesala del colapso. Barcelona: Libros del Lince, 2014.

VARGAS, G. Educación y desarrollo en los Andes. Frankfurt: Peter Lang, 2005.

VARGAS, G. Fundamentos educativos para la construcción de un estado inclusivo en Bolivia. En: Bolivien. Neue Wege und alte Gegensätze. Berlín: WVB, 2006. p. 327-362.

VARGAS, G.; VARGAS, J. P. El proyecto de Ley de Educación Boliviana “Avelino Siñani - Elizardo Pérez" ¿Fundamentos para la construcción de un socialismo comunitario? En: Libro de Actas del Seminario Internacional de Políticas Educativas Iberoamericanas. Barcelona: Universidad de Barcelona, 2010. p. 838-852. 


\section{PRENSA BOLIVIANA CONSULTADA:}

CAMBIO. Disponible en: <www.cambio.bo $>$.

EL CORREO DEL SUR. Disponible en: <www.correodelsur.com>.

EL DEBER: Disponible en: <www.eldeber.com.bo> .

EL DIARIO. Disponible en: <www.eldiario.net>.

LA RAZÓN. Disponible en: <www.la-razon.com>.

LOS TIEMPOS. Disponible en: <www.lostiempos.com>.

OPINIÓN. Disponible en: <www.opinión.com.bo>.

Texto recebido em 13 de outubro de 2014.

Texto aprovado em 13 de outubro de 2014. 